\title{
ANATOMÍA MACROSCÓPICA DE LA MUSCULATURA DEL MIEMBRO ANTERIOR DEL MONO MACHÍN BLANCO (Cebus albifrons)
}

\section{Macroscopic Anatomy of the Forelimb Musculature in the White Fronted Capuchin Monkey (Cebus albifrons)}

\author{
Nelly Cribillero $C h .^{1,2}$, Alberto Sato $S_{.}{ }^{1,3}$ y Miluska Navarrete $Z .^{1}$
}

\section{RESUMEN}

El objetivo del presente estudio fue identificar y describir las estructuras musculares del miembro anterior del mono Machín blanco (Cebus albifrons). Se utilizaron 4 monos machos adultos. Se emplearon las técnicas estándares de embalsamiento y se realizó la disección de las caras lateral y medial del miembro anterior. Se describen 42 músculos, de los cuales seis conforman la región del hombro, seis la región del brazo, 19 la región del antebrazo siendo 11 en el lado lateral y 8 en el lado medial, y 11 en la región de la mano.

Palabras clave: Cebus albifrons, mono Machín Blanco, miembro anterior, músculos

\section{AbSTract}

The objective of the present study was to identify and describe the muscular structures of the forelimb of the white fronted capuchin (Cebus albifrons). Four male adult specimens were used. The standard techniques for embalming were used and the dissection of the muscles was made in the lateral and medial side of the forelimb. It is described 42 muscles, of which six muscles were in the shoulder girdle, six muscles in the region of the upper arm, 19 muscles in the region of the forearm where 11 were located in the lateral side and eight in the medial side, and 11 muscles in the region of the hand.

Key words: Cebus albifrons, White Fronted Capuchin monkey, forelimb, muscles

\footnotetext{
${ }^{1}$ Laboratorio de Anatomía Animal y Fauna Silvestre, Facultad de Medicina Veterinaria, Universidad Nacional Mayor de San Marcos, Lima

${ }^{2}$ E-mail: giovanna06@yahoo.com

${ }^{3}$ E-mail: albertosato2000@yahoo.com
} 
Estudios sobre la anatomía macroscópica de los primates del Nuevo Mundo son escasos en comparación con los existentes sobre monos del Viejo Mundo, especialmente sobre la anatomía del mono Rhesus Macaca mulatta (Bast et al., 1961). En el Perú, se dispone de un reporte reciente sobre la anatomía de los músculos del miembro posterior y la cola en C. albifrons (Quevedo et al., 2009).

El estudio anatómico muscular aporta información relevante con relación a su función para las actividades del animal y constituye una herramienta importante en el conocimiento de los hábitos del animal (Aversi et al., 2005). Los monos han desarrollado características anatómicas únicas y morfología funcional que le permiten adaptarse a su ambiente (Turnquist y Hong, 1995). Los primates tienen una visión mejorada, gran desarrollo cerebral, y la habilidad de sujetar objetos con pies y manos les permiten trepar y manipular su ambiente; asimismo, el largo de los brazos y piernas varía, dependiendo si se desenvuelven sobre el suelo o en árboles (Ott, 2003). El machín blanco tiene una excepcional destreza manual, posee pulgares oponibles y una cola semiprensil (Robinson y Janson, 1987; Fortman et al., 2002).

El significado clínico de las características de la morfología de los miembros en primates no humanos es múltiple, incluyendo locaciones para venopuntura y biopsia de nervios periféricos. Las extremidades son morfológica y funcionalmente más similares a las extremidades humanas que a cualquier otro animal (Turnquist y Hong, 1995).

El presente estudio busca determinar las semejanzas y diferencias que puedan existir en la anatomía del miembro anterior del Cebus albifrons, con relación a la anatomía del miembro anterior de otros primates no humanos y humanos.

\section{Materiales y Métodos}

El estudio se realizó en el Laboratorio de Anatomía Animal de la Facultad de Medicina Veterinaria (FMV) de la Universidad Nacional Mayor de San Marcos (UNMSM), Lima. Se emplearon cuatro monos Machines blancos (Cebus albifrons) machos adultos, donados por el Patronato del Parque de las Leyendas.

Los monos fueron anestesiados y sacrificados por el personal veterinario del $\mathrm{Pa}$ tronato del Parque de las Leyendas, y remitidos al Laboratorio de Anatomía Animal, FMVUNMSM. Se practicó un corte en el rafe medio ventral del cuello, para exponer la arteria carótida común y la vena yugular externa. Se colocaron clamps arteriales en el extremo de cada vaso y se practicó un corte longitudinal de $0.5 \mathrm{~cm}$ para proceder al vaciamiento de sangre. El embalsamamiento se hizo con solución conservadora (formol al $12 \%$, acido fénico al 3\%, glicerina al $8 \%$ y agua csp 100\%), inyectada vía arteria carótida común, previa canulación, con una bomba aspirante-impelente (120 $\mathrm{mm}$ de $\mathrm{Hg})$, hasta su retorno por la vena yugular. Además, se hicieron múltiples infiltraciones en el cuerpo con formol al $20 \%$.

Se realizó la disección de las caras lateral y medial del hombro, brazo, antebrazo y mano. La descripción macroscópica de cada músculo (m.) se hizo desde el plano superficial al plano profundo y de lateral a medial. La nomenclatura de los músculos de los primates se hizo en base a la nomenclatura usada en el mono rhesus (Macaca mulatta) y a la Nómina Anatómica Veterinaria (ICVGAN, 2005).

\section{Resultados}

\section{Músculos del Hombro}

Supraespinoso. Presentó una sola porción. Se encontró debajo del $\mathrm{m}$. trapecio porción 
cervical y el m. atlantoescapular anterior.

- Origen: Fosa supraespinosa

- Inserción: Parte más alta de la tuberosidad mayor del húmero.

Infraespinoso. Se relacionó con el $\mathrm{m}$. redondo menor en la parte craneal, cubierto parcialmente en la porción distal por el $\mathrm{m}$. redondo mayor, y en su lado anterior cubierto parcialmente por el $\mathrm{m}$. deltoides porción espinosa.

- Origen: Fosa infraespinosa de la escápula.

- Inserción: Parte superior del borde posterior de la tuberosidad mayor del húmero.

Redondo mayor. Músculo fusiforme, corto y ancho, cubierto parcialmente por el músculo dorsal ancho en la parte inferior. En craneal limitó con el $\mathrm{m}$. tríceps cabeza larga.

- Origen: Ángulo caudal de la escápula y en el tercio inferior del borde lateral de la escápula.

- Inserción: Borde medial del húmero debajo del cuello quirúrgico.

Redondo menor. Se localizó a lo largo del borde caudal de la escápula.

- Origen: Borde caudal de la escápula.

- Inserción: Borde posterior de la extremidad distal de la tuberosidad mayor del húmero.

Subescapular. Músculo multipennado (en haces). Se extendió enteramente sobre la superficie medial de la escápula.

- Origen: Superficie medial de la escápula.

- Inserción: Tuberosidad menor del húmero.

Deltoides. Voluminoso. Presentó tres porciones: espinosa, acromial y clavicular, que se unen en la parte inferior formando un vértice.

- Origen: Porción espinosa: (también se le conoce como porción escapular). Espina de la escápula. Porción acromial: Acromión. Porción clavicular: Borde anterior de la clavícula.
- Inserción: Cara anterior del húmero en la porción distal de la tuberosidad deltoidea.

\section{Músculos del Brazo}

Tríceps braquial. Ancho, aplanado y grueso. Situado sobre la superficie, lateral y medial del húmero. Se dividió en 3 cabezas: larga, lateral y medial.

- Origen:

Cabeza larga: Borde caudal de la escápula, a través de una porción amplia donde llegan fibras musculares y fibras tendinosas.

Cabeza lateral: Lado póstero-lateral del húmero, en el tercio proximal en la línea tricipital. Se extiende hasta debajo de la inserción del redondo menor en el cuello anatómico (unión cabeza-cuello).

Cabeza medial: A lo largo del borde posterior del húmero y del borde lateral en la porción media distal.

- Inserción: Olécranon.

Braquial. Mediano y grueso. Se relacionó en craneal con el $\mathrm{m}$. bíceps braquial y en caudal con el m. tríceps cabeza lateral.

- Origen: Tercio medio y parte del tercio proximal en la cara lateral y medial del húmero.

- Inserción: Proceso coronoides del hueso cúbito.

Ancóneo. Pequeño, íntimamente relacionado al $\mathrm{m}$. tríceps y algunas veces se continuó (a) como extensión distal del m. tríceps.

- Origen: Epicóndilo lateral del húmero y borde lateral distal del húmero.

- Inserción: Olécranon hasta la cara lateral del cúbito proximal.

Coracobraquial. Pequeño. Presentó dos porciones: profunda y media.

- Origen:

Porción profunda: Tendón coracoide común y apófisis coracoides. 
Porción media: Tendón coracoide común, y apófisis coracoides, profundo al bíceps braquial cabeza corta.

- Inserción:

Porción profunda: Cuello del húmero, sobre la inserción del m. dorsal ancho.

Porción media: Parte media del lado medial del húmero, entre el $\mathrm{m}$. braquial y la cabeza medial del $\mathrm{m}$. tríceps.

Bíceps braquial. Grande, grueso y voluminoso. Se extendió a lo largo del cuerpo del húmero. Presentó dos porciones o cabezas: corta y larga.

- Origen:

Cabeza corta: Mediante un tendón situado entre la división del $\mathrm{m}$. coracobraquial. Se origina de la apófisis coracoides.

Cabeza larga: En la tuberosidad supraglenoidea.

- Inserción: Mediante un tendón que se inserta en la tuberosidad radial.

Tensor de la fascia antebraquial. Ancho y aplanado, de forma ovalada. No presentó tendón de inserción. Se encontró caudal al bíceps braquial.

- Origen: Músculo del dorsal ancho.

- Inserción: Fascia del olécranon y epicóndilo medial del húmero.

\section{Músculos del Antebrazo (Cara lateral)}

Braquiorradial. Músculo desarrollado. Cuerpo muscular ancho y plano. La porción proximal fue amplia y el tendón de inserción plano y corto (Fig. 1).

- Origen: Borde lateral del húmero, en el tercio distal, tabique intermuscular lateral y cresta epicondílea lateral del húmero.

- Inserción: Base de la apófisis estiloides del radio.

Extensor carpo radial largo. Alargado y menos ancho que el extensor carpo radial corto. Presentó un tendón largo y delgado.
Limitó en craneal con el $\mathrm{m}$. braquiorradial y en caudal con el $\mathrm{m}$. extensor carpo radial corto (Fig. 1).

- Origen: Borde lateral del tercio distal del húmero.

- Inserción: Superficie dorsal de la extremidad proximal del $2^{\text {do }}$ metacarpo.

Extensor carpo radial corto. Cuerpo ancho y alargado, tendón grueso y largo. Se encontró entre el $\mathrm{m}$. extensor carpo radial largo en craneal y el m. extensor digital común en caudal (Fig. 1).

- Origen: Parte superior del epicóndilo lateral del húmero.

- Inserción: Superficie dorsal de la extremidad proximal del $3^{\text {er }}$ metacarpo.

Extensor digital común. Alargado, ancho y aplanado. Presentó un tendón grueso que se dividió al llegar a la aponeurosis transversa dorsal del carpo. Se encontró entre el m. extensor carpo radial corto en craneal, y el m. extensor digital lateral en caudal. Se dividió en 8 tendones delgados (Fig. 1).

- Origen: Epicóndilo lateral del húmero.

- Inserción: Porción dorsal de la extremidad distal de la segunda falange de los dedos II, III, IV y V.

Extensor digital lateral. Alargado y delgado, separado por un tabique de fascia del $\mathrm{m}$. extensor digital común. Se localizó entre el $\mathrm{m}$. extensor digital común en craneal y el $\mathrm{m}$. extensor carpo cubital en caudal. El tendón pasó por debajo del ligamento transverso dorsal del carpo, y se dividió en dos tendones que se dirigen al IV y V dedo (Fig. 1).

- Origen: Epicóndilo lateral del húmero.

- Inserción: Superficie cubital de la articulación metacarpofalangiana del IV dedo. Superficie cubital del dorso de la falange media en la articulación interfalangiana proximal del $\mathrm{V}$ dedo.

Extensor carpo cubital. Aplanado y alargado. Limitó en craneal con el m. extensor digital lateral y en caudal con el borde lateral del 
hueso cúbito (Fig. 1).

- Origen: Epicóndilo lateral del húmero.

- Inserción: Superficie lateral de la base del $5^{\text {to }}$ metacarpo.

Supinador. Largo y aplanado con fibras tendinosas sobre su cara lateral. Se extendió sobre las caras anterior, lateral y posterior del radio. Está cubierto por el $\mathrm{m}$. extensor carpo radial corto y el $\mathrm{m}$. extensor digital común; en caudal se encuentró el $\mathrm{m}$. abductor del I dedo.

- Origen: Epicóndilo lateral del húmero y parte del borde lateral del cúbito.

- Inserción: Extremidad proximal del radio en sus caras anterior, lateral y posterior.

Abductor largo del pulgar (I dedo). Aplanado y alargado. El tendón de inserción no estuvo cubierto por el ligamento transverso dorsal del carpo (Fig. 1).

- Origen: Borde lateral del cúbito, porción proximal del borde lateral del radio y la membrana interósea.

- Inserción: Superficie radial de la base del $1^{\text {er }}$ metacarpo y en la parte superior del sesamoideo radial adyacente.

Extensor del I y II dedo. El nombre de este músculo está relacionado a su origen e inserción. Se localizó profundo al m. extensor carpo cubital, se relacionó por su borde caudal con el cúbito y por su borde craneal con el $\mathrm{m}$. abductor del I dedo. Cubrió parcialmente a los músculos extensor propio del II y III dedo. Músculo delgado y alargado. Se dividió en dos cuerpos musculares, una porción larga y una porción corta, y de cada cuerpo muscular salió un tendón. La porción larga se ubicó en craneal, presentó el tendón más largo y grueso que salió a la altura de la parte final del tercio medio del cúbito. La porción corta se encontró distal a la porción larga, y se continuó con un tendón delgado que salió a partir del tercio distal del cúbito. Este tendón se anastomosó con el tendón grueso de la porción larga a la altura del tercio proximal del $2^{\text {do }}$ metacarpo.
- Origen: Tercio medio y parte del tercio proximal del borde lateral del cúbito y la membrana interósea.

- Inserción: La porción larga en la superficie dorsal de la extremidad distal de la primera falange del I dedo. La porción corta en la superficie dorsal de la articulación metacarpofalangiana del II dedo.

Extensor propio del II dedo. Pequeño y delgado. Se encontró distal al $\mathrm{m}$. extensor del I y II dedo. Se localizó entre el $\mathrm{m}$. abductor del primer dedo en craneal y el $\mathrm{m}$. extensor propio del III dedo en caudal.

- Origen: Parte antero lateral del tercio distal del cúbito.

- Inserción: Superficie lateral de la I falange del II dedo.

Extensor propio del III dedo. Pequeño y delgado.

- Origen: Borde lateral del tercio distal del cúbito.

- Inserción: Extremidad media de la $1^{\mathrm{a}}$ falange del III dedo.

\section{Músculos del Antebrazo (Cara medial)}

Pronador redondo. Cilíndrico, ancho. Se localizó entre el $\mathrm{m}$. braquiorradial en craneal y el m. flexor carpo radial en caudal.

- Origen: Epicóndilo medial del húmero.

- Inserción: Borde posterior y lateral del tercio medio del radio.

Flexor carpo radial. Aplanado y de cuerpo ancho, tendón grueso y largo. Se localizó entre el $\mathrm{m}$. pronador redondo en craneal, y $\mathrm{m}$. palmar largo en caudal. Cubrió al m. flexor digital superficial.

- Origen: Epicóndilo medial del húmero y cápsula de la articulación del codo.

- Inserción: Extremidad proximal del $2^{\text {do }}$ metacarpo.

Palmar largo. Aplanado, delgado y alargado, con un tendón delgado y largo. El tendón 
se situó superficial al ligamento transverso palmar del carpo. Se localizó entre el $m$. flexor carpo radial en craneal y el $\mathrm{m}$. flexor carpo cubital en caudal.

- Origen: Epicóndilo medial del húmero.

- Inserción: El tendón llega y se divide en la aponeurosis palmar.

Flexor carpo cubital. Se localizó entre el $\mathrm{m}$. palmar largo en craneal y el hueso cúbito en caudal. Músculo grueso, aplanado y ancho. Presentó 2 porciones: humeral y cubital.

- Origen:

Porción humeral: epicóndilo medial del húmero.

Porción cubital: olécranon.

- Inserción: Carpo accesorio o hueso pisiforme.

Epitrochleo anconeus. Pequeño. Se extendió oblicuamente desde el epicóndilo medial del húmero hacia su inserción en el olécranon.

- Origen: Epicóndilo medial del húmero y ancóneo.

- Inserción: Olécranon.

Flexor digital superficial. Grueso de forma alargada. Presentó, además, una pequeña porción muscular más delgada en forma de cinta, cubierta por la porción más gruesa de este músculo.

- Origen: Epicóndilo medial del húmero.

- Inserción: Extremidad proximal del borde palmar de la $2^{\text {a }}$ falange de los dedos II, III, IV y V.

Flexor digital profundo. Músculo desarrollado con tres porciones: cubital, humeral y radial.

Porción cubital: La más grande de las tres porciones, alargado y con un tendón ancho y plano, se dirigió al IV y V dedo.

- Origen: Región lateral y palmar del cúbito.

Porción humeral: Menos desarrollada. Cuerpo muscular delgado y corto. Se continuó con un tendón que se unió a la porción radial en la extremidad proximal del radio.
- Origen: Epicóndilo medial del húmero.

Porción radial: Cuerpo muscular grueso y con fibras oblicuas. Se asentó sobre la superficie lateral y posterior del radio. Llevó tendones al I, II y III dedo.

- Origen: Lado medial y volar del radio y membrana interósea.

- Inserción de las tres porciones: Extremidad distal de la falange media de los cinco dedos.

Pronador cuadrado. Se localizó debajo del $\mathrm{m}$. flexor digital profundo, ocupando el tercio distal del antebrazo, del espacio interóseo.

- Origen: Borde posterior del tercio distal del cúbito.

- Inserción: Borde posterior del tercio distal del radio.

\section{Músculos de la mano}

Palmar corto. Músculo subcutáneo, pequeño y ancho, en forma de una banda. Se localizó cubriendo a los músculos palmares mediales por completo.

- Origen: Borde cubital de la aponeurosis palmar.

- Inserción: Borde lateral de la mano $\mathrm{y}$ en parte en el hueso pisiforme y fascia.

Abductor corto del pulgar. Se localizó craneal al m. flexor corto del pulgar, y cubrió al m. oponente del pulgar (I dedo).

- Origen: Ligamento transverso palmar del carpo (retináculo flexor), sesamoideo radial.

- Inserción: Base de la $1^{\mathrm{a}}$ falange del I dedo y parte en el $1^{\text {er }}$ metacarpo.

Oponente del pulgar. Se localizó debajo del $\mathrm{m}$. abductor corto del pulgar. Músculo delgado y corto, cubierto por el $\mathrm{m}$. abductor corto del pulgar y parcialmente por la cabeza superficial del m. flexor corto del pulgar (Fig. 2).

- Origen: Ligamento transverso palmar del carpo, sesamoideo radial y trapecio. 


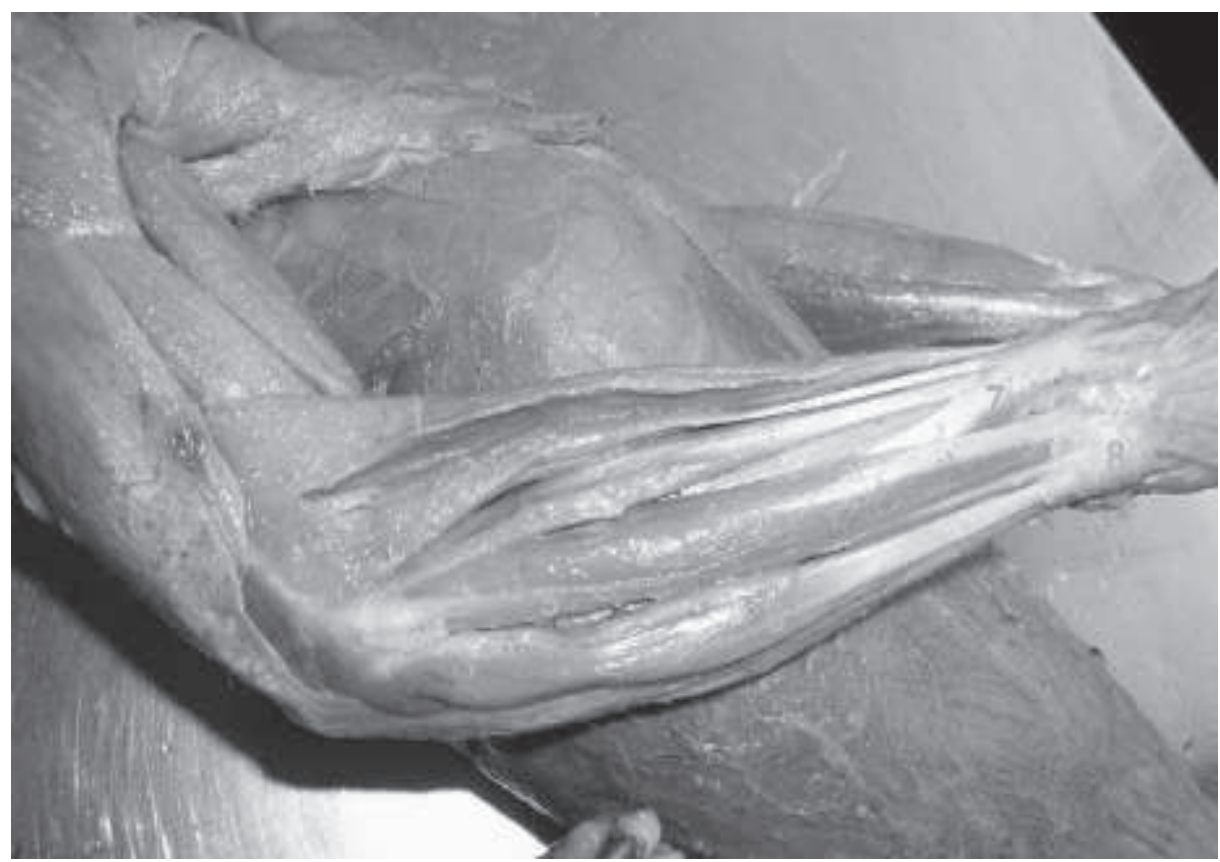

Figura 1. Músculos superficiales de la cara lateral de antebrazo del mono Cebus albifrons. 1: $\mathrm{m}$. braquioradial, $2: \mathrm{m}$. extensor carpo radial largo, 3: $\mathrm{m}$. extensor carpo radial corto, 4 : $\mathrm{m}$. extensor digital común, 5: $\mathrm{m}$. extensor digital lateral, 6: $\mathrm{m}$. extensor carpo cubital, 7: $\mathrm{m}$. abductor largo del pulgar, 8: ligamento transverso dorsal del carpo

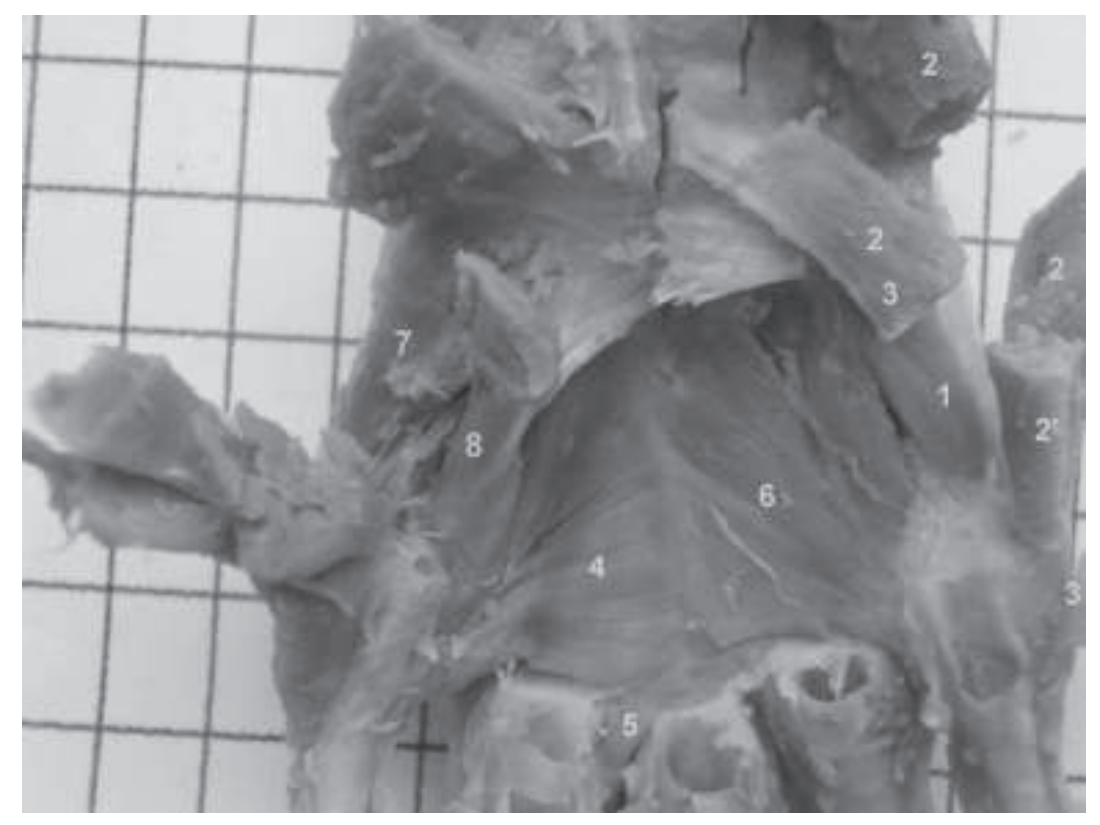

Figura 2. M. profundos de la cara palmar de la mano del mono Cebus albifrons. 1: m. oponente del $\mathrm{V}$ dedo, 2: $\mathrm{m}$. abductor del $\mathrm{V}$ dedo, 3: $\mathrm{m}$. flexor corto del $\mathrm{V}$ dedo, 4: $\mathrm{m}$. adductor del pulgar, 5: $\mathrm{m}$. contrahente del II dedo, 6: $\mathrm{m}$. contrahente del V dedo, 7: $\mathrm{m}$. oponente del pulgar, $8: \mathrm{m}$. flexor corto del pulgar cabeza profunda. 
- Inserción: Borde radial del $1^{\text {er }}$ metacarpo y en la base de la $1^{\text {a }}$ falange del I dedo.

Flexor corto del pulgar (I dedo): Presentó 2 cabezas: superficial y profunda. La cabeza profunda (Fig. 2) se dividió en 2 porciones: grande y pequeña. La porción grande fue más superficial y se unió a la base de la $1^{\text {a }}$ falange del pulgar sobre la cara radial del tendón del $\mathrm{m}$. flexor digital profundo; la porción pequeña, más profunda, se localizó parcialmente fusionada en su origen con las fibras proximales del $\mathrm{m}$. adductor del pulgar y se insertó con el $\mathrm{m}$. adductor del pulgar sobre la cara medial de la base de la $1^{\text {a }}$ falange del dedo pulgar.

- Origen:

Cabeza superficial: Ligamento transverso palmar del carpo.

Cabeza profunda: Hueso trapezoide y base del $2^{\text {do }}$ metacarpo.

- Inserción: Base de la primera falange del I ${ }^{\text {er }}$ dedo.

Adductor del pulgar. Músculo en forma de abanico (Fig. 2).

- Origen: Bases del $2^{\circ}$ y $3^{\text {er }}$ metacarpo, y cápsulas de la $2^{\mathrm{a}}$ y $3^{\mathrm{a}}$ articulación metacarpofalangianas.

- Inserción: Base de la primera falange del pulgar (I dedo).

Abductor del $V$ dedo. Presentó 2 cabezas: grande y pequeña. Se insertó mediante dos tendones desarrollados (Fig. 2).

- Origen:

Cabeza grande: Hueso pisiforme, borde cubital de la mano, y de la extensión palmar del ligamento transverso dorsal del carpo.

Cabeza pequeña: Ligamento transverso palmar del carpo.

- Inserción: Cápsula de la articulación metacarpofalangiana del $\mathrm{V}$ dedo, y base de la $1^{\text {a }}$ falange del $\mathrm{V}$ dedo.

Flexor corto del $V$ dedo. (Fig. 2).

- Origen: Ligamento transverso palmar del carpo.
- Inserción: Superficie cubital de la $1^{\text {a }}$ falange del $\mathrm{V}$ dedo, superficie dorsal del $\mathrm{V}$ dedo entre la $1^{\mathrm{a}}$ y $2^{\mathrm{a}}$ falange, $\mathrm{y}$ cápsula de la articulación metacarpofalangiana del $\mathrm{V}$ dedo.

Oponente del $V$ dedo. El más profundo de los músculos del V dedo (Fig. 2).

- Origen: Hueso ganchoso y por debajo del ligamento transverso palmar del carpo.

- Inserción: Superficie cubital de la diáfisis del $\mathrm{V}$ metacarpo.

Lumbricales. Fueron 4 músculos. El cuerpo muscular fue alargado y en forma de huso.

- Origen: Superficies laterales de los tendones del $\mathrm{m}$. flexor digital profundo. El $1^{\text {er }}$ y $2^{\circ}$ músculo lumbrical se origina de la cara anterior y borde lateral del tendón del flexor digital profundo del II y III dedo. El $3^{\circ} \mathrm{y}$ el $4^{\circ}$ se originan entre las caras adyacentes del tendón del $\mathrm{m}$. flexor digital profundo.

- Inserción: Tercio proximal de la superficie radial de las primeras falanges de los dedos II al V.

Contrahentes digitales. Presentó 2 músculos: el contrahente del II dedo y el contrahente del $\mathrm{V}$ dedo. Este último fue el más grande. Se originó de una sola porción desde el comienzo del tendón de origen y continuó a lo largo de la superficie cubital de la prolongación del tendón. Se extendió en forma de abanico. El contrahente del II dedo fue mucho más pequeño y en forma de una cinta delga$\mathrm{da}$, y a veces cubierto por el $\mathrm{m}$. aductor del I dedo.

- Origen:

Contrahente del V dedo: Bases del $2^{\circ}$ y $3^{\text {er }}$ hueso metacarpiano, trapezoide y hueso grande.

Contrahente del II dedo: Superficie radial de la extremidad distal del tendón del contrahente.

- Inserción:

Contrahente del V dedo: Superficie radial de la base de la $1^{a}$ falange del $\mathrm{V}$ dedo. 
Contrahente del II dedo: Superficie cubital de la base de la $1^{\text {a }}$ falange del II dedo.

Interóseos. Fueron 11 músculos, pequeños y alargados. Siete se encontraron en la superficie palmar y fueron los interóseos palmares, uno por cada lado del $2^{\circ}, 3^{\circ}$ y $4^{\circ}$ metacarpo, y el séptimo sobre la superficie radial del $5^{\circ}$ metacarpo. Los otros cuatro músculos fueron los interóseos dorsales y ocuparon los espacios interóseos dorsales. Se localizaron en la superficie radial del $2^{\circ}$ metacarpo, la superficie radial y cubital del $3^{\text {er }}$ metacarpo, y la superficie cubital del $4^{\circ}$ metacarpo.

- Origen: Bases del $2^{\circ}, 3^{\circ}, 4^{\circ}$ y $5^{\circ}$ huesos metacarpianos y de los ligamentos carpales adyacentes.

- Inserción: Base de la primera falange del dedo respectivo.

\section{Discusión}

En el estudio descriptivo de los músculos del miembro anterior del machín blanco se han observado varias similitudes con lo descrito en el mono rhesus (Macaca mulatta) (Bast et al., 1961), en el mono Machín negro (Cebus apella) (Aversi et al., 2005, 2006) y el hombre (Latarjet y Ruiz, 2004); sin embargo, también se han encontrado algunas diferencias en algunos músculos de la región del antebrazo y mano.

El m. coracobraquial en el machín blanco estuvo dividido en dos porciones: profunda y media, ambas con un mismo origen y diferente inserción, mientras que en el mono rhesus se considera dos músculos coracobraquiales: $\mathrm{m}$. coracobraquial profundo y $\mathrm{m}$. coracobraquial medio (Bast et al., 1961), siendo la porción profunda pequeña y delgada, y la porción media desarrollada. A diferencia, en el hombre, el coracobraquial consta de una sola porción desarrollada y comparte el tendón de origen con la cabeza corta del bíceps braquial (Latarjet y Ruiz, 2004).
El m. tensor de la fascia antebraquial se observó desarrollado en el machín blanco, al igual que en el mono rhesus, en el cual recibe el nombre de $\mathrm{m}$. dorso epitrochlearis. Este músculo está normalmente ausente en el hombre, pero es encontrado en todos los prosimios y usualmente en gorilas (Bast et al., 1961).

En el machín blanco, el m. extensor digital lateral presentó una porción muscular, la cual se dividió en dos tendones que se dirigieron al IV y V dedo. En el mono rhesus está considerado como dos músculos diferentes ( $\mathrm{m}$. extensor propio del IV dedo y $\mathrm{m}$. extensor propio del V dedo) (Bast et al., 1961), que tienen el mismo origen y las mismas inserciones que en el machín blanco. En el hombre, al igual que en el machín negro, se encuentra sólo el $\mathrm{m}$. extensor del meñique (V dedo) (Aversi et al., 2005), en donde el tendón de inserción se anastomosa con el tendón del extensor digital común (Latarjet y Ruiz, 2004); lo que no sucede en el machín blanco, el machín negro (Aversi et al., 2005) y el mono rhesus (Bast et al., 1961), donde el tendón se inserta independientemente, sin anastomosarse con el tendón del extensor digital común.

En el machín blanco se observó un músculo delgado y poco desarrollado en el grupo de los extensores, que se insertó en los dedos I y II, es considerado como extensor del I y II dedo, presentó dos porciones musculares, larga y corta, las cuales desprenden dos tendones que se dirigieron hacia el I y II dedo, y se comunicaron a nivel del tercio proximal del $2^{\circ}$ metacarpo; en cambio, en el hombre y el mono rhesus existe el $\mathrm{m}$. extensor largo del I dedo (Bast et al., 1961) y, además, solo en el hombre está presente el $\mathrm{m}$. extensor corto del I dedo (Latarjet y Ruiz, 2004).

El m. extensor propio del III dedo está presente en el mono machín blanco y rhesus (Bast et al., 1961), pero ausente en el hombre (Latarjet y Ruiz, 2004). Asimismo, el m. flexor carpo cubital presenta dos cabezas: 
humeral y cubital, en el machín blanco y el hombre (Latarjet y Ruiz, 2004). En el mono rhesus y el machín negro no se consideran dos cabezas pero sí se describen dos orígenes: en el epicóndilo medial del húmero y en el olécranon (Bast et al., 1961; Aversi et al., 2006).

El m. epitrochleo anconeus se observó en el machín blanco, y en el rhesus está relacionado genéticamente al flexor carpo cubital (Bast et al., 1961), pero no está presente en el hombre (Latarjet y Ruiz, 2004).

El flexor digital superficial del machín blanco presentó una sola porción muy desarrollada, que tuvo su origen en el epicóndilo medial del húmero, profundo al músculo (Bast et al., 1961), mientras que en el hombre, consta de tres cabezas: humeral, cubital y radial (Latarjet y Ruiz, 2004), y en el machín negro se describen dos cabezas: humeral y radial (Aversi et al., 2006).

En el machín blanco, el m. flexor digital profundo presentó tres cabezas: cubital, humeral y radial, mientras que en el mono rhesus se considera la cabeza radial, la cubital y una accesoria, la cual recibe las fibras del flexor digital superficial, y llevan tendones del I al V dedo (Bast et al., 1961). En el hombre no se consideran cabezas y se divide en cuatro lengüetas musculares de donde salen tendones a los II, III, IV y V dedos (Latarjet y Ruiz, 2004).

Los músculos contrahentes se observan en el machín blanco y el mono rhesus, mientras que en el hombre están ausentes (Bast et al., 1961). El machín blanco presentó dos músculos contrahentes, el del II y del V dedo, de la misma forma, origen e inserción que en el mono rhesus, a excepción del origen del contrahente del $\mathrm{V}$ dedo que en el mono rhesus se origina de dos cabezas, del tendón del contrahente y de su extensión en el $4^{\circ}$ metacarpo (Bast et al., 1961). El mono rhesus presenta, además, un contrahente del
IV dedo, que sale del inicio del tendón contrahente sobre el $3^{\text {er }}$ metacarpo, lado ulnar del mismo tendón hasta la base de la primera falange del IV dedo (Bast et al., 1961).

\section{Conclusiones}

- Los músculos del miembro anterior del mono machín blanco (Cebus albifrons) son similares a lo descrito en el mono rhesus (Macaca mulatta), y en forma similar al hombre y al machín negro $(C$. apella).

- En el miembro anterior del machín blanco (C. albifrons) se ha observado y descrito 42 músculos, mientras que el mono rhesus se describen 44 músculos y en el hombre 40 músculos.

\section{Literatura Citada}

1. Aversi T, Pires $R$, Da Silveira P, Aversi R, Silva Z. 2005. Anatomia dos músculos extensores superficiais do antebrazo do macaco Cebus. Int J Morphol (Supl 23) 1:5.

2. Aversi T, Da Silveira P, Pires R, Aversi $R$, Silva Z. 2006. Anatomia dos músculos flexores superficiais do antebrazo do macaco Cebus. Biosci J 22: 139-144.

3. Bast T, Kopel B, Christensen K. 1961. The anatomy of the Rhesus monkey (Macaca mulatta). $2^{\text {nd }}$ ed. New York: Hafner Publishing. 383 p.

4. Fortman J, Hewett T, Bennet B. 2002. The laboratory: Non human primate. USA: CRC Press. 266 p.

5. [ICVGAN] International Committee on Veterinary Gross Anatomical Nomenclature. 2005. Nomina Anatómica Veterinaria. 5a ed. Hannover: ICVGAN. 165 p.

6. Latarjet M, RuizA. 2004. Anatomía humana. $4^{a}$ ed. Buenos Aires: Médica Panamericana. 594 p. 
7. Ott J. 2003. Other primates excluding great apes. In: Fowler M, Miller R(eds). Zoo and wild animal medicine. USA: Saunders. p 346-355.

8. Quevedo M, Cisneros J, Navarette M, Torres J, Sato A. 2009. Descripción anatómica de los músculos del miembro posterior y cola del mono machín blanco (Cebus albifrons). Rev Inv Vet, Perú 20 (en prensa).

9. Robinson J, Janson C. 1987. Capuchins, squirrel monkeys, and atelines: sociological convergence with old world primates. In: Smuts BB, Chenty DL, Seyfarth RB, Wrangham RW, Struhsaker TT (eds). Primate Societies. USA: University of Chicago Press. p 69-82.

10. Turnquist J, Hong N. 1995. Functional morphology. In: Bennett B, Abee CR, Henrickson R (eds). Nonhuman primates in biomedical research: Biology and management. USA: Academic Press. $p$ 49-76. 